\title{
Haplotypes of the imprinted insulin gene are associated with size for gestational age and umbilical cord IGF-II levels Ronald Adkins*1, Julia Krushkal2 ${ }^{\text {, Chad Klauser }}{ }^{4}$, Everett Magann5, Grant Somes ${ }^{2}$, John Fain ${ }^{3}$ and John Morrison ${ }^{4}$
}

\author{
Address: ${ }^{1}$ Department of Pediatrics, University of Tennessee Health Science Center, Memphis, TN 38103, USA, ${ }^{2}$ Department of Preventive \\ Medicine, University of Tennessee Health Science Center, Memphis, TN 38103, USA, ${ }^{3}$ Department of Molecular Sciences, University of Tennessee \\ Health Science Center, Memphis, TN 38103, USA, ${ }^{4}$ Obstetrics and Gynecology, University of Mississippi Medical Center, Jackson, MS 39216, USA \\ and ${ }^{5}$ Obstetrics and Gynecology, Naval Medical Center at Portsmouth, Portsmouth, VA 23708, USA \\ Email: Ronald Adkins* - radkins1@utmem.edu \\ * Corresponding author
}

from UT-ORNL-KBRIN Bioinformatics Summit 2008

Cadiz, KY, USA. 28-30 March 2008

Published: 8 July 2008

BMC Bioinformatics 2008, 9(Suppl 7):P9 doi:10.1 |86/|47| |-2 I05-9-S7-P9

This abstract is available from: http://www.biomedcentral.com//47/-2105/9/S7/P9

(c) 2008 Adkins et al; licensee BioMed Central Ltd.

\section{Objective}

To test the association between haplotypes in the insulin - insulin-like growth factor 2 (IGF2) locus and both risk of small for gestational age birth and umbilical cord IGFII levels.

\section{Subjects}

207 pairs of healthy African-American full-term, newborn and mothers from Memphis, Tennessee and Jackson, Mississippi.

\section{Methods}

Associations of individual SNPs and inferred haplotypes with risk of small for gestational age (SGA) birth were tested using logistic regression, and mean umbilical cord IGF-II levels were compared by ANOVA. The risk of SGA and differences in cord IGF-II were also compared according to the parental origin of haplotypes.

\section{Results}

In newborns three INS SNPs exhibited significant $(\mathrm{p}<$ 0.01 ) association with reduced SGA risk. Two of these SNPs were significantly associated with umbilical cord IGF-II levels. In the mothers, the alternate SNP alleles were associated with reduced risk of SGA. No maternal SNPs were associated with umbilical cord IGF-II levels.
When analyzed according to parental origin of haplotypes, paternally transmitted haplotypes were significantly associated with risk of SGA and umbilical cord IGFII levels, but maternally transmitted haplotypes were not significantly associated.

\section{Conclusion}

Newborn genotypes for polymorphisms near the 5 ' end of the insulin gene are significantly associated with size for gestational age and umbilical cord IGF-II levels, with a major effect due to the paternally inherited allele, which is preferentially expressed due to imprinting. There is some evidence that complementary haplotypes confer reduced risk of SGA in mothers and newborns.

\section{Acknowledgements}

This work was supported by grants from the NICHD, Le Bonheur Children's Medical Center and the Children's Foundation Research Center of Memphis. 